\title{
Patterning of dielectric nanoparticles using dielectrophoretic forces generated by ferroelectric polydomain films
}

\author{
P. Mokrý, 1, a) M. Marvan, ${ }^{2}$ and J. Fousek ${ }^{1}$ \\ ${ }^{1)}$ Institute of Mechatronics and Computer Engineering, Technical University of Liberec, CZ-46117 Liberec, \\ Czech Republic \\ ${ }^{2)}$ Faculty of Mathematics and Physics, Charles University, 18000 Praha 8, Czech Republic
}

(Dated: 21 April 2022)

\begin{abstract}
A theoretical study of a dielectrophoretic force, i.e. the force acting on an electrically neutral particle in the inhomogeneous electric field, which is produced by a ferroelectric domain pattern, is presented. It has been shown by several researchers that artificially prepared domain patterns with given geometry in ferroelectric single crystals represent an easy and flexible method for patterning dielectric nanoobjects using dielectrophoretic forces. The source of the dielectrophoretic force is a strong and highly inhomogeneous (stray) electric field, which exists in the vicinity of the ferroelectric domain walls at the surface of the ferroelectric film. We analyzed dielectrophoretic forces in the model of a ferroelectric film of a given thickness with a lamellar $180^{\circ}$ domain pattern. The analytical formula for the spatial distribution of the stray field in the ionic liquid above the top surface of the film is calculated including the effect of free charge screening. The spatial distribution of the dielectrophoretic force produced by the domain pattern is presented. The numerical simulations indicate that the intersection of the ferroelectric domain wall and the surface of the ferroelectric film represents a trap for dielectric nanoparticles in the case of so called positive dielectrophoresis. The effects of electrical neutrality of dielectric nanoparticles, free charge screening due to the ionic nature of the liquid, domain pattern geometry, and the Brownian motion on the mechanism of nanoparticle deposition and the stability of the deposited pattern are discussed.
\end{abstract}

\section{INTRODUCTION}

A well-known fact follows from the Maxwell theory that the inhomogeneous electromagnetic field induces a force even on electrically neutral particles. However, for a long time there has been no attention on how this phenomenon could be practically applied. A new impetus in this field was provided in a 1951 paper by Pohl $^{1}$ where he studied the influence of inhomogeneous electrostatic field on the motion of small plastic particles suspended in insulating dielectric fluids. Pohl named this phenomenon dielectrophoresis (DEP) and it was shown in his 1978 monograph $^{2}$ that DEP forces can be usefully used for the manipulation of small particles. This book, in fact, motivated studies of other possibilities to use the electric field for trapping or controlling the motion of small particles.

At the same time, in 1980s, a new field of nanotechnology emerged and DEP has become an important and intensively studied tool for the manipulation with nanoparticles. Examples to be mentioned here are the DEP assembly of nanowires from nanoparticle suspensions ${ }^{3}$, DEP precise positioning of carbon nanotubes ${ }^{4}$, particle separation using $\mathrm{DEP}^{5-7}$, DEP formation of nanodroplets ${ }^{8}$ or nanofibers ${ }^{9}$, etc. In all the aforementioned applications, the inhomogeneous electric field, which is essential for the existence of DEP forces, is achieved by properly arranged electrodes. The crucial point related to nanotechnology is that, when there

${ }^{a)}$ Electronic mail: pavel.mokry@tul.cz is a wish to work with nanoparticles, it is necessary to deposit very thin electrodes which are very close to each other. This task can be obviously managed ${ }^{10}$ but, in fact, it is usually technologically demanding. In addition, a fixed set of electrodes unfortunately does not allow flexible changes in the nanoparticle pattern geometry, which may be necessary in some advanced applications.

In order to overcome disadvantages of the fixed electrode approach, attention of researchers has been drawn to answer the question: would it be more useful to use an inhomogeneous field existing above the surface of a ferroelectric film with a domain pattern? The interest of scientists in this question has been stimulated by a great advance that has recently been achieved in a precise writing of ferroelectric domains of nanometer dimensions. Several methods for nanoscale domain control in ferroelectric films have been developed by several groups of researchers. Classical examples of domain engineering to be mentioned here are the domain poling with patterned electrodes ${ }^{11-13}$, domain patterning by scanning probe microscopy ${ }^{14-18}$, and focused electron or ion beam domain engineering ${ }^{19-21}$. Details about advanced nanoscale domain engineering methods can be found in the recent review paper by $\mathrm{Li}$ and Bonnell ${ }^{22}$. The aforementioned methods make it possible to prepare arbitrary ferroelectric domain patterns in a flexible way.

In fact, there exist two different means to use ferroelectric domains for nanoparticles patterning. The first approach is based on the use of domain-specific chemical reactions and it is called ferroelectric lithography ${ }^{23,24}$. Using this method, the direct assembly of virus particles has been realized by Dunn et al. ${ }^{25}$ The second approach is based on the use of physical DEP forces that are pro- 
duced by bound charges at the surface of the ferroelectric film with a domain pattern, which has been recently reported by $\mathrm{Ke}^{26}$ et al. and Grilli et al. ${ }^{27}$.

It is the second approach that has motivated the theoretical analysis presented below, where we will address the study of the DEP forces produced by lamellar $180^{\circ}$ domain pattern. In Sec. II, we first present the details of our model of a ferroelectric film with $180^{\circ}$ domain domain pattern. Section III presents a straightforward calculation of the electrostatic potential and the electric field produced by the ferroelectric domain pattern. We will cover the general configuration of the domain pattern including the effect of free charge screening as well as two cases of a special interest. First, the domain pattern with wide domains compared to the thickness of the ferroelectric film (Sec. III A) and the system with a dense domain pattern (Sec. IIIB). In Sec. IV, calculation and numerical simulations of the DEP forces produced domain patterns in four systems with different configurations are presented and compared. Section V presents a brief discussion of the effect of electrical neutrality of dielectric nanoparticles on the mechanism of nanopatterning (Sec. VA), the effect of free charge screening on the inhomogeneous field and the value of DEP force (Sec. V B), the role of domain pattern geometry on the value of the DEP force produced by the ferroelectric domain pattern (Sec. V C), the role of the Brownian motion on the stability of the nanoparticle patterns (Sec. VD), and, finally, the estimation of the values of important numerical parameters that are essential in the design of a ferroelectric system used for patterning of nanoparticles (Sec. VE).

\section{GEOMETRY AND MATERIAL PROPERTIES OF THE FERROELECTRIC FILM}

Figure 1 shows the geometry of the considered model of a ferroelectric film with a lamellar $180^{\circ}$ ferroelectric domain pattern crossing the film thickness $d$. The space above the top surface of the film is filled with an ionic liquid of permittivity $\varepsilon_{m}$ and with a given concentration of free charge carriers. The bottom surface of the ferroelectric film is attached to the ground electrode. We consider that the top and bottom surfaces of the film are perpendicular to the ferroelectric axis $z$ of the attached Cartesian coordinate system. The axis $x$ of the coordinate system is perpendicular to the system of $180^{\circ}$ domain walls. Using the "hard ferroelectric" approximation, we express the electric displacement of the ferroelectric as a sum of the linear dielectric response of the crystal lattice to the electric field and the constant spontaneous polarization $P_{0}$ (whose orientation differs from domain to domain):

$$
\begin{aligned}
D_{x} & =\varepsilon_{0} \varepsilon_{a} E_{x}, \\
D_{z} & =\varepsilon_{0} \varepsilon_{c} E_{z} \pm P_{0},
\end{aligned}
$$

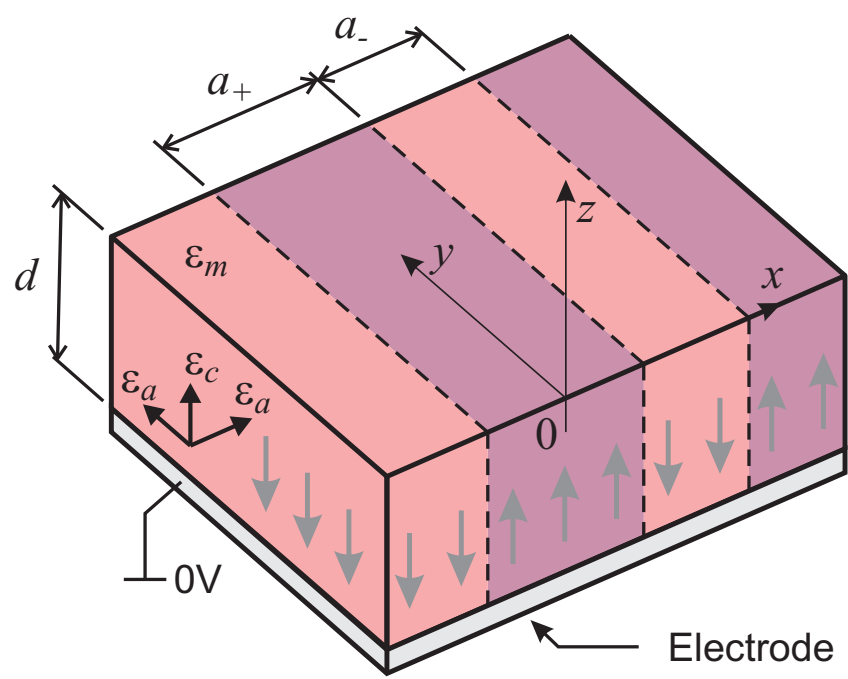

FIG. 1. Geometry of the ferroelectric film of the thickness $d$ with the $180^{\circ}$ domain pattern. Gray arrows indicate the orientation of the vector of spontaneous polarization in the film. Dashed lines indicate positions of the $180^{\circ}$ domain walls. Symbols $a_{+}$and $a_{-}$stand for the widths of domains where the vector of the spontaneous polarization is oriented along and against the orientation of the $z$-axis of the attached coordinate system, respectively. Symbols $\varepsilon_{a}$ and $\varepsilon_{c}$ stand for the relative permittivity tensor components of the ferroelectric, symbol $\varepsilon_{m}$ stands for the permittivity of the liquid.

where $\varepsilon_{c}$ and $\varepsilon_{a}$ are the components of the permittivity tensor of the crystal lattice in the directions parallel and perpendicular to the ferroelectric axis, respectively, and $\varepsilon_{0}$ is the vacuum permittivity. We consider that the vector of spontaneous polarization is perpendicular to the film surfaces and its magnitude $P_{0}$ is constant within each domain. For given widths $a_{+}$and $a_{-}$of domains, where the vector of the spontaneous polarization is oriented along and against the direction of the $z$-axis of the attached coordinate system, respectively, we define the average (net) spontaneous polarization of the ferroelectric layer $P_{N}=P_{0}\left(a_{+}-a_{-}\right) /(2 a)$, where $a=\left(a_{+}+a_{-}\right) / 2$ is the domain spacing.

The abrupt change of the spontaneous polarization to zero at the interface of the ferroelectric film and the ionic liquid yields the appearance of a bound charge of the surface density $\sigma_{b}(x)$, which is the source of the spatially nonuniform electric field. The spatial distribution of the bound charge due to the discontinuous change of the normal component of the spontaneous polarization at the ferroelectric film surface in the direction of the $x$ axis can be written in the form:

$$
\sigma_{b}(x)=P_{N}+\sum_{n=1}^{\infty} \frac{4 P_{0}}{\pi n} \sin \left[\frac{n \pi}{2}\left(1+\frac{P_{N}}{P_{0}}\right)\right] \cos n k x,
$$

where $k=\pi / a$. 


\section{ELECTROSTATIC POTENTIAL PRODUCED BY THE DOMAIN PATTERN}

In this section we present the calculation of the electrostatic potential produced by the ferroelectric domain pattern. Since the dielectric properties of the ferroelectric film and the ionic liquid are quite different, we use the following symbols $\varphi$ and $\varphi_{f}$ for the electrostatic potential in the ionic liquid (for $z>0$ ) and the ferroelectric film (for $0>z>-d$ ), respectively. In our analysis, we consider the general situation where the ionic liquid above the top surface of the ferroelectric film contains free charge carriers of given concentration. In such a situation, the electric field is screened by the free charge carriers and vanishes within some typical distance from the surface of the ferroelectric film. In order to calculate the spatial distribution of the electrostatic potential in the system, we adopt the Debye-Hückel theory ${ }^{28}$ (DHT) and the aforementioned functions for the electrostatic potential should satisfy: first, the following partial differential equations of DHT and electrostatics

$$
\begin{aligned}
\frac{\partial^{2} \varphi}{\partial x^{2}}+\frac{\partial^{2} \varphi}{\partial z^{2}}-\frac{\varphi}{\lambda_{D}^{2}} & =0 \\
\varepsilon_{a} \frac{\partial^{2} \varphi_{f}}{\partial x^{2}}+\varepsilon_{c} \frac{\partial^{2} \varphi_{f}}{\partial z^{2}} & =0
\end{aligned}
$$

where

$$
\lambda_{D}=\sqrt{\frac{\varepsilon_{0} \varepsilon_{m} k_{B} T}{2 N_{A} e^{2} I}}
$$

is the Debye screening length, $k_{B}$ is the Boltzmann constant, $T$ is the thermodynamic temperature, $N_{A}$ is the Avogadro number, $e$ is the electron charge, $I=$ $(1 / 2) \sum_{i} c_{i} Z_{i}^{2}$ is the ionic strength of the liquid, where $c_{i}$ and $Z_{i}$ are the molar concentration and the charge number of the $i$-th particular ion in the liquid.

Second, the boundary conditions for the continuity of the electrostatic potential

$$
\begin{aligned}
\varphi & =\varphi_{f} & & \text { at } z=0, \\
\varphi_{f} & =0 & & \text { at } z=-d .
\end{aligned}
$$

Third, the boundary condition for the continuity of the tangential component of the electric field

$$
\frac{\partial \varphi}{\partial x}=\frac{\partial \varphi_{f}}{\partial x} \quad \text { at } z=0
$$

Fourth, the boundary condition for the continuity of the normal component of the electric displacement

$$
-\varepsilon_{m} \frac{\partial \varphi}{\partial z}+\varepsilon_{c} \frac{\partial \varphi_{f}}{\partial z}=\frac{\sigma_{b}(x)}{\varepsilon_{0}} \quad \text { at } z=0
$$

It is a straightforward task to show that the the following functions in the form of Fourier series represent the solution of the electrostatic problem given by Eqs. (3):

$$
\begin{gathered}
\varphi=\frac{d P_{N}}{\varepsilon_{0} \varepsilon_{c} \xi_{D}} e^{-\frac{\hat{z}}{k \lambda}}+\frac{C_{E}}{k} \sum_{n=1}^{\infty} \sin \left(\frac{n \pi}{2}+n \hat{p}\right) \times \\
\frac{e^{-n \hat{z} \eta_{D}(n)}}{n^{2}\left[\eta_{D}(n)+g \operatorname{coth} n R\right]} \cos (n \hat{x}), \\
\varphi_{f}=\frac{P_{N}(d+\hat{z} / k)}{\varepsilon_{0} \varepsilon_{c} \xi_{D}}+\frac{C_{E}}{k} \sum_{n=1}^{\infty} \sin \left(\frac{n \pi}{2}+n \hat{p}\right) \times \\
\frac{\sinh (n R+n c \hat{z})}{n^{2}\left[\eta_{D}(n) \sinh n R+g \cosh n R\right]} \cos (n \hat{x}),
\end{gathered}
$$

where $\hat{x}=k x, \hat{z}=k z, \hat{p}=\pi P_{N} /\left(2 P_{0}\right), c=\sqrt{\varepsilon_{a} / \varepsilon_{c}}$, $g=c \varepsilon_{c} / \varepsilon_{m}, R=c k d$, and

$$
\begin{aligned}
C_{E} & =\frac{4 P_{0}}{\pi \varepsilon_{0} \varepsilon_{m}}, \\
\xi_{D} & =1+\frac{\varepsilon_{m} d}{\varepsilon_{c} \lambda_{D}}, \\
\eta_{D}(n) & =\sqrt{1+\left(n k \lambda_{D}\right)^{-2}} .
\end{aligned}
$$

In the special case of the absence of free charge carriers in the liquid, i.e. infinite value of the Debye screening length $\lambda_{D}$, the values of parameters $\xi_{D}$ and $\eta_{D}(n)$ for all $n \geq 1$ tend to 1 and the solution of the electrostatic problem given by Eqs. (3) reduces down to the form that is already available in literature ${ }^{29}$, however in the special case of the ferroelectric film in a vacuum, i.e. for $\varepsilon_{m}=1$.

Equations (4) represent the exact solution of the electrostatic potential produced by the domain pattern. For the sake of clearer graphical presentation, it is convenient to use the normalized value $\Phi$ of the electrostatic potential: $\Phi=\varphi\left(k / C_{E}\right)$. In the following subsections, we will present two special situations in the system with the absence of free charge carriers, i.e. $\lambda_{D} \gg a$, in which the above expressions can be further simplified.

\section{A. Systems with thin ferroelectric films and wide domains}

The first situation is considered for the systems with an isolating dielectric liquid where the thickness of the ferroelectric film $d$ is smaller than the average distance between the domain walls $a$, i.e. $R \ll 1$. In addition, it is reasonable to consider that the permittivity of ferroelectric film is much larger than the permittivity of the dielectric liquid, i.e. $g \gg 1$. In this case, the factors $(1+g \operatorname{coth} n R)$ in the denominator of the leading terms in Eq. (4a) are dominated by $g \operatorname{coth} n R$ and it is quite acceptable to approximate $(1+g \operatorname{coth} n R)$ by $g(1+\operatorname{coth} n R)$. Using the Euler formula $e^{i z}=\cos z+i \sin z$, using the sum and difference formulas for the trigonometric functions, and using the following formula:

$$
\sum_{n=1}^{\infty} \frac{e^{n(\xi+i \eta)}}{n^{2}(1+\operatorname{coth} n R)}=\frac{1}{2}\left[\operatorname{Li}_{2}\left(e^{\xi+i \eta}\right)-\operatorname{Li}_{2}\left(e^{\xi-2 R+i \eta}\right)\right]
$$




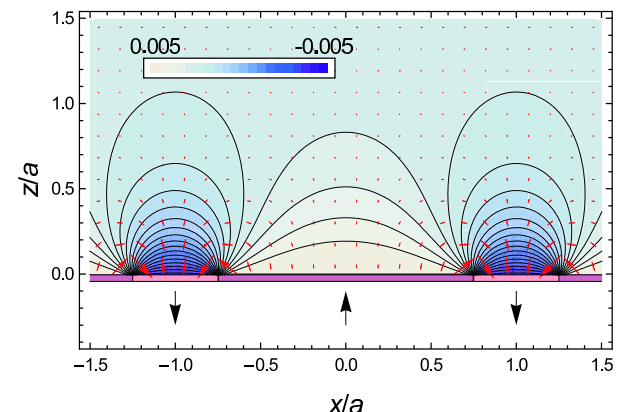

a) $R=0.1$ and $k \lambda_{D}=10^{3}$

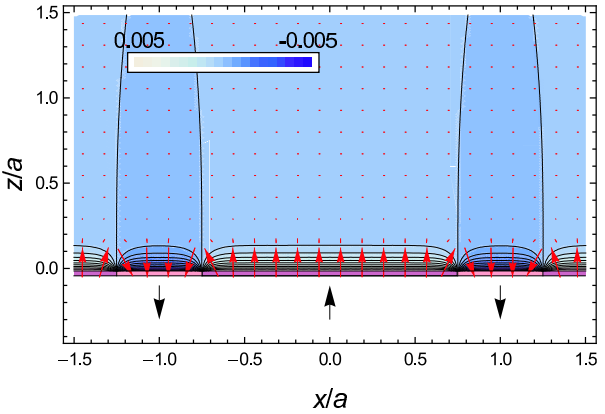

c) $R=0.1$ and $k \lambda_{D}=0.2$

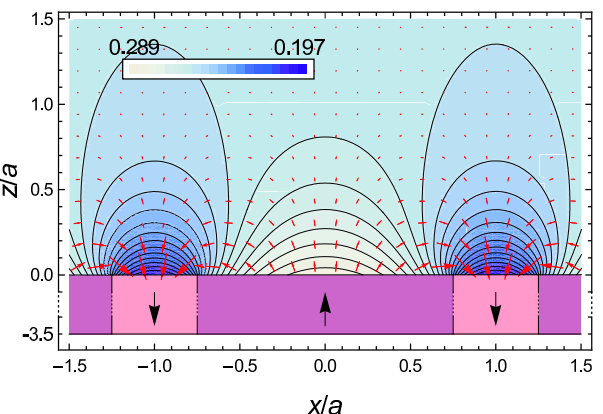

b) $R=10$ and $k \lambda_{D}=10^{3}$

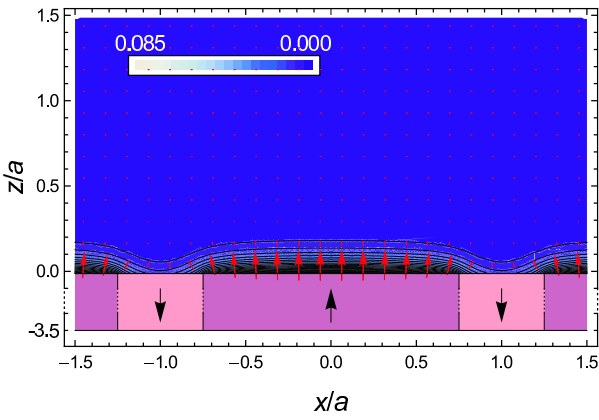

d) $R=10$ and $k \lambda_{D}=0.2$

FIG. 2. Contour plot of the electrostatic potential produced by the domain pattern in the system with a thin ferroelectric film and wide domains, $R=0.1,(\mathrm{a}, \mathrm{c})$ and with a dense domain pattern, $R=10$, (b, d). In addition, situations with the absence of free charge carriers, $k \lambda_{D}=10^{3}$, (a, b), and with free charge screening, $k \lambda_{D}=0.2$, (c, d), are compared. The electrostatic potential is normalized to the constant $\left(C_{E} / k\right)$ given by Eq. (4c). Red arrows represent the inhomogeneous electric field produced by the domain pattern. The following values of material parameters were considered $\hat{p}=0.5, g=15.8$. Note the difference in the peak values of the normalized electrostatic potential and in the normalized electrostatic potential gradient in the vicinity of the domain walls in the cases (a, c) and (b, d).

(5) with wide domains. It should be noted that in the case of systems where the permittivity of the dielectric liquid, $\varepsilon_{m}$, is approximately equal to $\sqrt{\varepsilon_{c} \varepsilon_{a}}$, i.e. $g \approx 1$,

where $\operatorname{Li}_{\mathrm{s}} z=\sum_{n=1}^{\infty} z^{n} / n^{s}$ is the polylogarithm function, the electrostatic potential in the dielectric medium can be expressed in a form:

$$
\varphi^{(w)}=\frac{C_{E}}{k} \Phi^{(w)}\left(\frac{\pi x}{a}, \frac{\pi z}{a}, \frac{\pi d}{2 a} \sqrt{\frac{\varepsilon_{a}}{\varepsilon_{c}}}, \frac{\pi P_{N}}{2 P_{0}}\right),
$$

where the function $\Phi^{(w)}$ stands for the sum in Eq. (4a)

$$
\begin{array}{r}
\Phi^{(w)}(\hat{x}, \hat{z}, R, \hat{p})=\frac{\hat{p} R}{2 g}+\frac{1}{8 g}[f(\hat{x}, \hat{z}, \hat{p})+\quad(6 \mathrm{~b}) \\
f(-\hat{x}, \hat{z}, \hat{p})-f(\hat{x}, \hat{z}+2 R, \hat{p})-f(-\hat{x}, \hat{z}+2 R, \hat{p})]
\end{array}
$$

and

$$
f(\xi, \eta, \zeta)=i\left\{\operatorname{Li}_{2}\left[-i e^{-\eta-i(\xi+\zeta)}\right]-\operatorname{Li}_{2}\left[i e^{-\eta-i(\xi-\zeta)}\right]\right\} .
$$

The superscript ${ }^{(w)}$ indicates that the functions are expressed in the approximation of the thin ferroelectric film the above expressions Eqs. (6) equal exactly those in Eq. (4a).

The electric field in the liquid produced by the ferroelectric domain pattern with wide domains can be expressed in a straightforward way:

$$
\begin{aligned}
E_{x}^{(w)} & =-\frac{\partial \varphi^{(w)}}{\partial x}= \\
- & C_{E} \Phi_{\hat{x}}^{(w)}\left(\frac{\pi x}{a}, \frac{\pi z}{a}, \frac{\pi d}{2 a} \sqrt{\frac{\varepsilon_{a}}{\varepsilon_{c}}}, \frac{\pi P_{N}}{2 P_{0}}\right),
\end{aligned}
$$

$$
\begin{aligned}
E_{z}^{(w)} & =-\frac{\partial \varphi^{(w)}}{\partial z}= \\
- & C_{E} \Phi_{\hat{z}}^{(w)}\left(\frac{\pi x}{a}, \frac{\pi z}{a}, \frac{\pi d}{2 a} \sqrt{\frac{\varepsilon_{a}}{\varepsilon_{c}}}, \frac{\pi P_{N}}{2 P_{0}}\right),
\end{aligned}
$$

where

$$
\Phi_{\hat{x}}^{(w)}(\hat{x}, \hat{z}, R, \hat{p})=\frac{1}{8 g} \log \frac{[\cosh \hat{z}-\sin (\hat{x}-\hat{p})][\cosh (\hat{z}+2 R)+\sin (\hat{x}+\hat{p})]}{[\cosh \hat{z}+\sin (\hat{x}+\hat{p})][\cosh (\hat{z}+2 R)-\sin (\hat{x}-\hat{p})]},
$$




$$
\begin{aligned}
& \Phi_{\hat{z}}^{(w)}(\hat{x}, \hat{z}, R, \hat{p})=-\frac{1}{4 g} \arctan \frac{2 \cos \hat{p}\left(e^{\hat{z}} \cos \hat{x}+\sin \hat{p}\right)}{e^{\hat{z}}\left(e^{\hat{z}}+2 \cos \hat{x} \sin \hat{p}\right)-\cos 2 \hat{p}}+ \\
& \frac{1}{4 g} \arctan \frac{2 \cos \hat{p}\left(\cos \hat{x}+e^{-\hat{z}-2 R} \sin \hat{p}\right)}{2 \sin \hat{p}[\cos \hat{x}+\cosh (\hat{z}+2 R) \sin \hat{p}]+2 \cos ^{2} \hat{p} \sinh (\hat{z}+2 R)} .
\end{aligned}
$$

In the derivation of Eqs. (8a), we have used the property of the polylogarithm function $\partial\left(\mathrm{Li}_{2} z\right) / \partial z=-\log (1-$ $z) / z$ and the definition of the logarithm in a complex plane $\log z=\log |z|+i \arg z$.

\section{B. Systems with dense domain patterns}

The second situation, for which it is possible to write down a closed formula for the electrostatic potential and the components of the electric field, is the ferroelectric film with a dense domain pattern, i.e. the systems where the thickness of the ferroelectric film $d$ is larger than the average distance between the domain walls $a$, i.e. $R \gg 1$. In this case, the terms $\operatorname{coth} n R$ in the denominator of the leading terms in Eq. (4a) can be replaced by 1 and, thus, it is quite acceptable to approximate $(1+g \operatorname{coth} n R)$ by $(1+g)$. Following the same algebraic procedures as in the previous subsection, the electrostatic potential in the dielectric liquid can be expressed in a form:

$$
\varphi^{(d)}=\frac{C_{E}}{k} \Phi^{(d)}\left(\frac{\pi x}{a}, \frac{\pi z}{a}, \frac{\pi P_{N}}{2 P_{0}}\right),
$$

where the function $\Phi^{(d)}$ stands for the sum in Eq. (4a)

$$
\Phi^{(d)}(\hat{x}, \hat{z}, \hat{p})=\frac{\hat{p} R}{2 g}+\frac{1}{4(1+g)}[f(\hat{x}, \hat{z}, \hat{p})+f(-\hat{x}, \hat{z}, \hat{p})]
$$

The superscript ${ }^{(d)}$ indicates that the functions are expressed in the approximation of the dense domain pattern.

The electric field in the dielectric liquid produced by the dense ferroelectric domain pattern can be expressed in a straightforward way:

$$
\begin{aligned}
& E_{x}^{(d)}=-\frac{\partial \varphi^{(d)}}{\partial x}=-C_{E} \Phi_{\hat{x}}^{(d)}\left(\frac{\pi x}{a}, \frac{\pi z}{a}, \frac{\pi P_{N}}{2 P_{0}}\right) \\
& E_{z}^{(d)}=-\frac{\partial \varphi^{(d)}}{\partial z}=-C_{E} \Phi_{\hat{z}}^{(d)}\left(\frac{\pi x}{a}, \frac{\pi z}{a}, \frac{\pi P_{N}}{2 P_{0}}\right)
\end{aligned}
$$

where

$$
\begin{aligned}
& \Phi_{\hat{x}}^{(d)}(\hat{x}, \hat{z}, \hat{p})=\frac{1}{4(1+g)} \log \frac{\cosh \hat{z}-\sin (\hat{x}-\hat{p})}{\cosh \hat{z}+\sin (\hat{x}+\hat{p})} . \\
& \Phi_{\hat{z}}^{(d)}(\hat{x}, \hat{z}, \hat{p})=-\frac{1}{2(1+g)} \times \\
& \arctan \frac{2 \cos \hat{p}\left(e^{\hat{z}} \cos \hat{x}+\sin \hat{p}\right)}{e^{\hat{z}}\left(e^{\hat{z}}+2 \cos \hat{x} \sin \hat{p}\right)-\cos 2 \hat{p}}
\end{aligned}
$$

\section{Simulation of the electrostatic potential and electrophoretic force}

Figures $2 \mathrm{a}$ and $2 \mathrm{~b}$ show the spatial distribution of the electrostatic potential $\varphi$ given by Eq. (4a) and the vectors of the electric field produced by the periodic domain pattern in the dielectric liquid in the two aforementioned situations characterized by the absence of the free charge carriers in the liquid medium (i.e. with a large value of the Debye screening length $k \lambda_{D}=10^{3}$ ). Figure 2a shows the system with a thin ferroelectric film and wide domains, $R=0.1$, and Figure $2 \mathrm{~b}$ shows the system with a dense domain pattern, $R=10$, (b). The electrostatic potential $\varphi$ is normalized to the constant $\left(C_{E} / k\right)$ given by Eq. (4c) and, thus, the contour plots correspond to the spatial distributions of the dimensionless functions $\Phi$ given by Eq. (6b) and (9b). Figures $2 \mathrm{c}$ and $2 \mathrm{~d}$ present the effect of the inhomogeneous electric field screening by free charge carriers in the ionic liquid (i.e. the small value of the Debye screening length compared to the domain spacing, $\left.k \lambda_{D}=0.2\right)$ in the systems with the same geometry as in Figs. 2a and 2b. In the bottom part of the figure, there are indicated the positions of the $180^{\circ}$ domain walls and the thickness of the ferroelectric film. Red arrows represents the vectors of the inhomogeneous electric field produced by the domain pattern and they are proportional to the electrophoretic (EP) force, i.e. the Coulomb force acting on charged particles.

If we consider an approximately spherical particle of a diameter $r$ with free charges on its surface of a surface density $\sigma$, the electrophoretic force acting on such a particle is given by the classical formula:

$$
F_{e p}=-4 \pi r^{2} \sigma \nabla \varphi
$$

With use of the notation adopted in this Article, Eq. (12) can be rewritten in the form:

$$
\begin{aligned}
F_{e p} & =-4 \pi r^{2} \sigma\left(C_{E} / k\right) \nabla \Phi \\
& =-C_{e p} \widehat{\nabla} \Phi,
\end{aligned}
$$

where

$$
C_{e p}=4 \pi r^{2} \sigma C_{E}
$$

and the symbol $\widehat{\nabla}$ stands for the gradient operator in the $\hat{x}_{i}$-coordinate system, where $\hat{x}_{i}=k x_{i}$.

In the numerical calculation of the normalized electrostatic potential, we considered the values of the ionic liquid permittivity $\varepsilon_{m}=3$ and the in-plane and out-of plane permittivity of the ferroelectric $\varepsilon_{a}=84$ and $\varepsilon_{a}=29$, respectively, which corresponds to the dielectric properties 


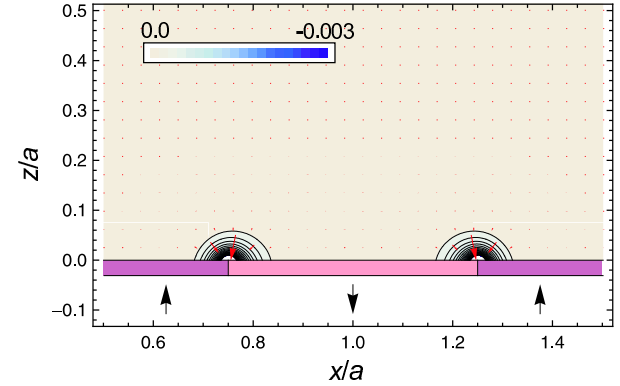

a) $R=0.1$ and $k \lambda_{D}=10^{3}$

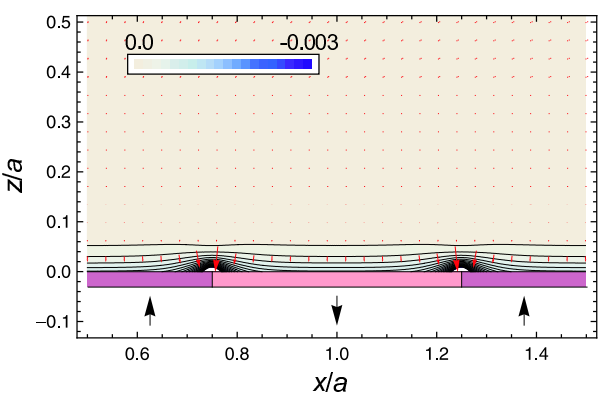

c) $R=0.1$ and $k \lambda_{D}=0.2$

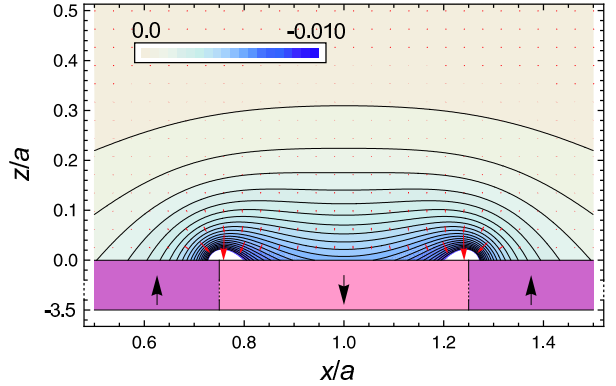

b) $R=10$ and $k \lambda_{D}=10^{3}$

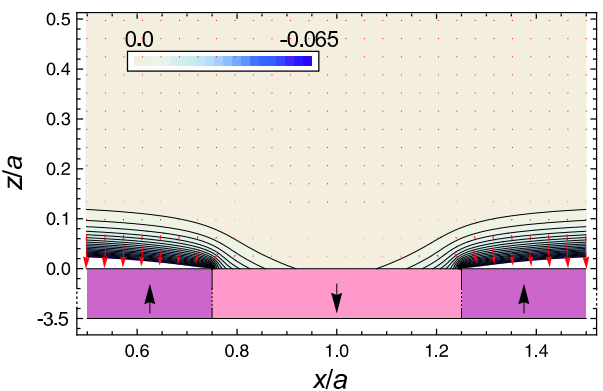

d) $R=10$ and $k \lambda_{D}=0.2$
FIG. 3. Contour plot of the positive DEP force potential produced by the domain pattern in the system with a thin ferroelectric film and wide domains, $R=0.1$, (a, c) and with a dense domain pattern, $R=$ 10, (b, d). In addition, situations with the absence of free charge carriers, $k \lambda_{D}=10^{3},(\mathrm{a}, \mathrm{b})$, and with free charge screening, $k \lambda_{D}=0.2$, (c, d), are compared. The potential is normalized to $C_{d e p}$. Red arrows represents the DEP force produced by the domain pattern. The following values of material parameters were considered $\hat{p}=0.5, g=15.8$. Note the difference in the peak values of the normalized dielectrophoretic force potential in the vicinity of the domain walls in the cases $(a, c)$ and $(b, d)$, which is controlled only by the geometry of the domain pattern. of lithium niobate ${ }^{30,31}$. The three aforementioned values of permittivity give the value of the parameter $g=15.8$. Finally, the non-neutral domain pattern was considered with $\hat{p}=0.5$, which corresponds to $P_{N} / P_{0}=0.32$. It is noticeable to see the difference in the peak values of the normalized electrostatic potential -0.005 and 0.005 in Fig. 2a; and 0.197 and 0.289 in Fig. 2b, which is not controlled by the values of spontaneous polarization $P_{0}$ but by the ratio of the ferroelectric film thickness over the domain spacing $d / a$, which is proportional to the value of the parameter $R$. The similar effect of the value of $R$ can bee seen on the normalized electrostatic potential gradient in the vicinity of the domain walls in Figs. 2a and $2 \mathrm{~b}$.

\section{DIELECTROPHORETIC FORCE}

The dielectrophoretic force $F_{d e p}$ that acts on approximately spherical particle of a diameter $r$ and the dielectric constant $\varepsilon_{p}$ in the inhomogeneous electric field $E$ is given by the classical formula ${ }^{1}$ :

$$
F_{\text {dep }}=K_{\text {dep }} \nabla\left(E_{x}^{2}+E_{z}^{2}\right),
$$

where

$$
K_{d e p}=2 \pi r^{3} \varepsilon_{m} \varepsilon_{0} \frac{\varepsilon_{p}-\varepsilon_{m}}{\varepsilon_{p}+2 \varepsilon_{m}} .
$$

It should be noted that depending on the permittivity values of the medium and particles, the situation characterized by $\varepsilon_{p}>\varepsilon_{m}$, i.e. $K_{d e p}>0$, is called positive dielectrophoresis and the situation, when $\varepsilon_{p}<\varepsilon_{m}$, i.e. $K_{\text {dep }}<0$, is called negative dielectrophoresis.

With use of the notation adopted in this Article, Eq. (14a) can be rewritten in the form:

$$
\begin{aligned}
F_{d e p} & =K_{d e p} C_{E}^{2} \nabla\left(\Phi_{\hat{x}}^{2}+\Phi_{\hat{z}}^{2}\right) \\
& =K_{d e p} C_{E}^{2} k \widehat{\nabla}\left(\Phi_{\hat{x}}^{2}+\Phi_{\hat{z}}^{2}\right) .
\end{aligned}
$$

For the purpose of the graphical presentation of spatial distribution of the DEP forces generated by the domain pattern, it is convenient to introduce the DEP force potential by formula $F_{d e p}=-\widehat{\nabla} U_{d e p}$. Thus, the function $U_{d e p}$ can be expressed in the form

$$
U_{d e p}=-C_{d e p}\left(\Phi_{\hat{x}}^{2}+\Phi_{\hat{z}}^{2}\right),
$$

where

$$
C_{d e p}=\frac{32 P_{0}^{2} r^{3}\left(\varepsilon_{p}-\varepsilon_{m}\right)}{a \varepsilon_{0} \varepsilon_{m}\left(\varepsilon_{p}+2 \varepsilon_{m}\right)} .
$$

Figures 3a-d show the spatial distribution of the dielectrophoretic field potential $U_{d e p}$ given by Eq. (16a) and the vectors of the dielectrophoretic force produced by the periodic domain pattern in the ionic liquid in the same four situations as in Figs. 2. Figures 3a and 3c show the systems with a thin ferroelectric film and wide domains, $R=0.1$. On the other hand, Figures $3 \mathrm{~b}$ and $3 \mathrm{~d}$ show the systems with a dense domain pattern, $R=10$. The dielectrophoretic field potential $U_{d e p}$ is normalized to the constant $C_{d e p}$ given by Eq. (16b) and, thus, the contour plots correspond to the spatial distributions of the dimensionless functions $-\left(\Phi_{\hat{x}}^{2}+\Phi_{\hat{z}}^{2}\right)$. Figures $3 \mathrm{a}$ and $3 \mathrm{~b}$ 
show the systems with a dielectric liquid and a low concentration of free charge carriers, which corresponds to the large value of the Debye screening length, $k \lambda_{D}=10^{3}$. On the contrary, Figs. 3c and 3d show the systems, where the inhomogeneous electric field is screened due to free charge carriers in the ionic liquid and where the Debye screening length is smaller than the domain spacing, i.e. $k \lambda_{D}=0.2$. It is noticeable to see the difference in the peak values of the normalized dielectrophoretic field potential in Figs. 3a and 3c, and 3b and 3d, respectively. Again the point is that the peak values are not controlled by the values of spontaneous polarization $P_{0}$ but by the ratio of the ferroelectric film thickness over the domain spacing $d / a$, which is proportional to the value of the parameter $R$.

\section{v. DISCUSSION AND CONCLUSIONS}

Theoretical results presented in the above Sections indicate qualitative features that play the key role in the decoration of nanoparticles using polydomain ferroelectric films. In the following subsections, we will briefly discuss (i) the effect of electrical neutrality of dielectric nanoparticles on the mechanism of nanopatterning, (ii) the effect of free charge screening on the inhomogeneous field and the value of DEP force, (iii) the role of domain pattern geometry on the value of the DEP force produced by the ferroelectric domain pattern, (iv) the role of the Brownian motion on the stability of the nanoparticle patterns, and, finally, (v) we will estimate the values of important numerical parameters that are essential in the design of a ferroelectric system used for patterning of nanoparticles.

\section{A. The role of electroneutrality of dielectric nanoparticles}

Since the electrical neutrality of nanoparticles is rarely the case in real experimental situations, it is necessary to compare the EP and DEP forces and to analyze the conditions that controls switching between the EP and DEP mechanisms of nanoparticle deposition.

Figure 4 shows the comparison of the EP and DEP force dependences on the ratio $x / a$ in the system with a thin ferroelectric film and wide domains, $R=0.1$ (solid line), and in the system with a dense domain pattern, $R=10$ (dashed line), at the distance $z=0.01 a$ from the surface of the ferroelectric film. The EP force value is normalized to $C_{e p}$ given by Eq. (13b). DEP force value is normalized to $C_{d e p}$ given by Eq. (16b) and, therefore, corresponds to the positive DEP force. It is seen that in the case of the EP mechanism, the $x$-component of the EP force is zero in the middle of each domain and the potentially charged nanoparticles would be preferentially deposited in the middle of those domains that have the opposite charge than the charged nanoparticle. On the contrary, the $x$-component of the DEP force is zero at

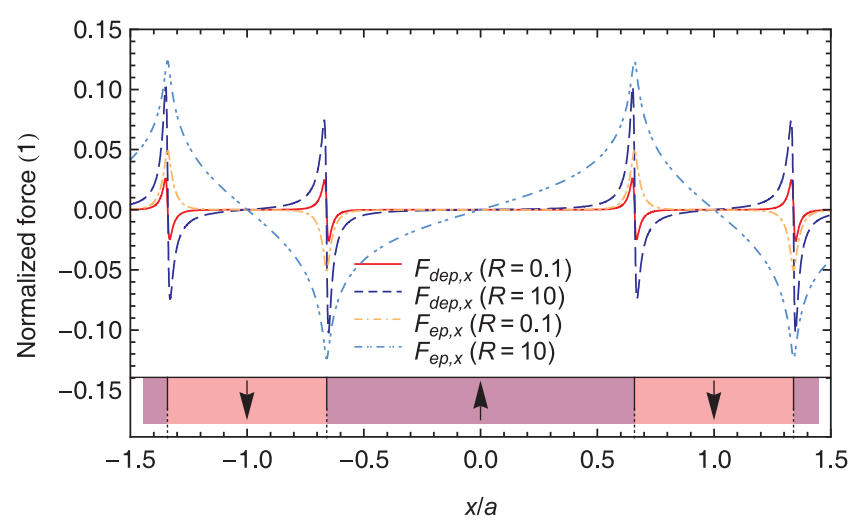

FIG. 4. Comparison of the $x$-component of the EP and DEP force dependences on the ratio $x / a$ in the system with a thin ferroelectric film and wide domains, $R=0.1$ (solid line), and in the system with a dense domain pattern, $R=10$ (dashed line), at the distance $z=0.01 a$ from the surface of the ferroelectric film. The EP force value is normalized to $C_{e p}$ given by Eq. (13b). DEP force value is normalized to $C_{d e p}$ given by Eq. (16b) and, therefore, corresponds to the positive DEP force. Note the qualitative difference between the EP and DEP force spatial distribution.

the domain wall position, abruptly increases with the increasing distance from the domain wall and reaches its maximum $F_{x, \max }$ at the distance $\Delta x_{\max }$ from the domain wall. It is seen that the reduction of the thickness of the film by a factor of 100, the peak values of the DEP force $x$-component a smaller by a factor of 10 . It is essential that the area of the intersection of the domain wall with the surface of the ferroelectric film represents a trap for nanoparticles in the case of a positive dielectrophoresis.

Figure 4 shows that the peak normalized values of the EP and DEP forces are of the same order of magnitude. Therefore, one should compare the parameters $C_{e p}$ and $C_{d e p}$ to get the critical value of the surface charge that controls the switching between the EP and DEP nanoparticle deposition mechanism. As a result, the DEP mechanism of deposition is dominant over the EP mechanism, when the surface charge density of the nanoparticles is much smaller than the critical value:

$$
\sigma \ll \sigma_{c r i t}=\frac{2 P_{0} r\left(\varepsilon_{p}-\varepsilon_{m}\right)}{a\left(2 \varepsilon_{m}+\varepsilon_{p}\right)} .
$$

Table I presents numerical values of materials parameters considered in our theoretical analysis for lithium tantalate $\left(\mathrm{LiTaO}_{3}\right)$, lithium niobate $\left(\mathrm{LiNbO}_{3}\right)$, barium titanate $\left(\mathrm{BaTiO}_{3}\right)$, and lead titanate $\left(\mathrm{PbTiO}_{3}\right)$ that can be prepared in a form of single crystal samples. In our numerical estimates, we further adopt parameters of materials that have been used previously in experiments by $\mathrm{Ke}$ et $a l{ }^{26}$, In particular, we consider the use of lithium niobate single crystal with the domain spacing $a=5 \mu \mathrm{m}$, polystyrene nanoparticles with dielectric constant $\varepsilon_{p}=2.7$ and radius $r=65 \mathrm{~nm}$, and dodecane liquid 
TABLE I. Material parameters (spontaneous polarization $P_{0}$, permittivity tensor components in the direction parallel and perpendicular to the direction of the spontaneous polarization $\varepsilon_{c}$ and $\varepsilon_{a}$, respectively, and the pyroelectric coefficient $\gamma_{S}$ at the room temperature) of selected ferroelectric materials: $\mathrm{LiTaO}_{3}{ }^{31,32}, \mathrm{LiNbO}_{3}{ }^{30,31}, \mathrm{BaTiO}_{3}{ }^{33,34}$, and $\mathrm{PbTiO}_{3}{ }^{35}$.

\begin{tabular}{|c|c|c|c|c|c|}
\hline Parameter & Unit & $\mathrm{LiTaO}_{3}$ & $\mathrm{LiNbO}_{3}$ & $\mathrm{BaTiO}_{3}$ & $\mathrm{PbTiO}_{3}$ \\
\hline$P_{0}$ & $\mathrm{Cm}^{-2}$ & 0.55 & 0.75 & 0.26 & 0.76 \\
\hline$\varepsilon_{c}$ & 1 & 44 & 29 & 188 & 66 \\
\hline$\varepsilon_{a}$ & 1 & 53 & 84 & 3600 & 124 \\
\hline$\gamma_{S}$ & $\mu \mathrm{Cm}^{-2} \mathrm{~K}^{-1}$ & 230 & 40 & 200 & 380 \\
\hline
\end{tabular}

with $\varepsilon_{m}=2$, the critical value of the nanoparticle surface charge is $\sigma_{\text {crit }} \approx 0.002 \mathrm{C} \mathrm{m}^{-2}$.

\section{B. The role of free charge screening}

Since the nanoparticle deposition experiments are carried out in liquid media, the presence of free charge carriers in the liquid cannot be completely avoided and sometimes it is even induced intentionally. It means that the effect of finite Debye screening length on the values of EP and DEP forces should be carefully analyzed. At first let us consider the simplest case of water. At room temperature, the permittivity of water is about 80 and the ionic strength equals $I=10^{-4} \mathrm{~mol} \mathrm{~m}^{-3}$, which yields the typical value of the Debye screening length $\lambda_{D} \approx 1 \mu \mathrm{m}$. In the case of nonpolar dielectric liquids, such as pentanoic acid or silicone oil, the value of the Debye screening length can be of several orders of magnitude larger.

Figure 2 clearly shows that the inhomogeneous electric field essentially decays within the distance of about $2 a$ from the surface of the ferroelectric film. This means that the decoration of nanoparticles using DEP forces should not be affected by free charge carriers in the systems where the Debye screening length is larger than the domain period $2 a$. From this condition, one can calculate the critical ionic strength of the liquid medium:

$$
I<I_{c r i t}=\frac{\varepsilon_{0} \varepsilon_{m} k_{B} T}{8 N_{A} e^{2} a^{2}}
$$

Considering the experiments by Grilli $^{27}$ with pentanoic acid $\varepsilon_{m}=2.66$ and the value of domain spacing $a=100 \mu \mathrm{m}$, the Eq. (18) yields the value $I_{\text {crit }}=$ $7.710^{-7} \mathrm{~mol} \mathrm{~m}^{-3}$.

\section{The role of domain pattern geometry}

Both Figs. 2 and 3 clearly indicate an essential role of the geometry of the domain pattern in the numerical value of the dielectrophoretic force produced by the bound charges of the surface of the ferroelectric film with a domain pattern. To get a clear insight into the role of domain pattern geometry on the DEP force, Fig. 5 shows the maximum value of the $x$-component of the DEP force and the position of this maximum, which is expressed in the distance from the domain wall and measured as a fraction of the domain spacing $a$, were calculated as a function of the parameter $R$ for three distances $z$ from the surface of the ferroelectric film. Figure 5 indicates that there is a qualitative difference in the aforementioned dependencies for values of $R$ smaller and greater than one. In the systems with a thin ferroelectric film and wide domains, which are characterized by $R<1$, the maximum value of the $x$-component of the DEP force is decreasing polynomially with a decreasing value of the parameter $R$. On the other hand, the systems with a dense ferroelectric domain pattern, which is characterized by $R>1$, the maximum of the DEP force is insensitive to the value or $R$. It should be noted that the maximum values of the DEP force $x$-component at the given distance $z=\hat{z} / k$ from the surface of the ferroelectric film are normalized to $C_{d e p}$ and they are, therefore, dominantly controlled by the geometry of the domain pattern.

\section{The role of Brownian motion}

In order to achieve a stable pattern of nanoparticles, the DEP force that acts to move the nanoparticle into the trap must overcome the forces that acts on that particle due to thermal movements of surrounding medium molecules, i.e. the forces that are responsible for the Brownian motion. The role of Brownian motion in DEP trapping of particles has been already analyzed by several authors ${ }^{36,37}$. Here we adopt the results achieved by Hughes and Morgan ${ }^{37}$ where the limit for particle trapping is estimated by comparing the velocities that are induced by DEP force with that of the Brownian motion. Result of this analysis yield the formula for the estimated critical minimal particle radius that is needed for successful trapping of a particle: ${ }^{37}$

$$
r>r_{\text {crit }}=\left(\frac{10 k_{B} T}{\pi \varepsilon_{m} \varepsilon_{0} K \Delta L\left|\nabla E^{2}\right|}\right)^{1 / 3},
$$

where

$$
K=\frac{\varepsilon_{p}-\varepsilon_{m}}{\varepsilon_{p}+2 \varepsilon_{m}}
$$

is the Clausius-Mossotti factor, $\Delta L$ is typical distance over which the field gradient $\nabla E^{2}$ has a given minimal value (see the inset in Fig. $5 \mathrm{~b}$ ), $k_{B}$ is the Boltzmann constant and $T$ is the thermodynamic temperature. In the notation of this Article, the above formula can be rewritten in the form:

$$
r_{c r i t}=\left(\frac{10 k_{B} T}{\pi \varepsilon_{m} \varepsilon_{0} K C_{E}^{2} S(\hat{z}, g, R)}\right)^{1 / 3},
$$

where

$$
S(\hat{z}, g, R)=\widehat{\Delta L}\left|\widehat{\nabla}_{\hat{x}}\left(\Phi_{\hat{x}}^{2}+\Phi_{\hat{z}}^{2}\right)\right|_{\mathrm{rms}}
$$




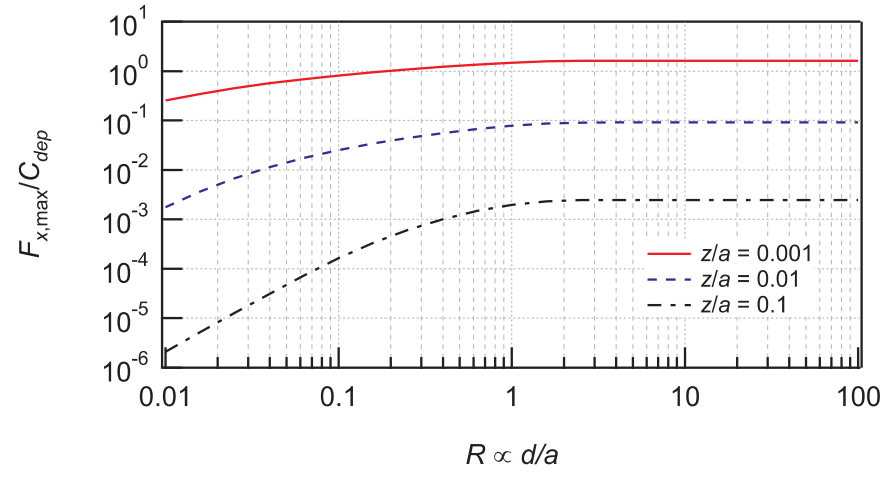

a) DEP force maximum value

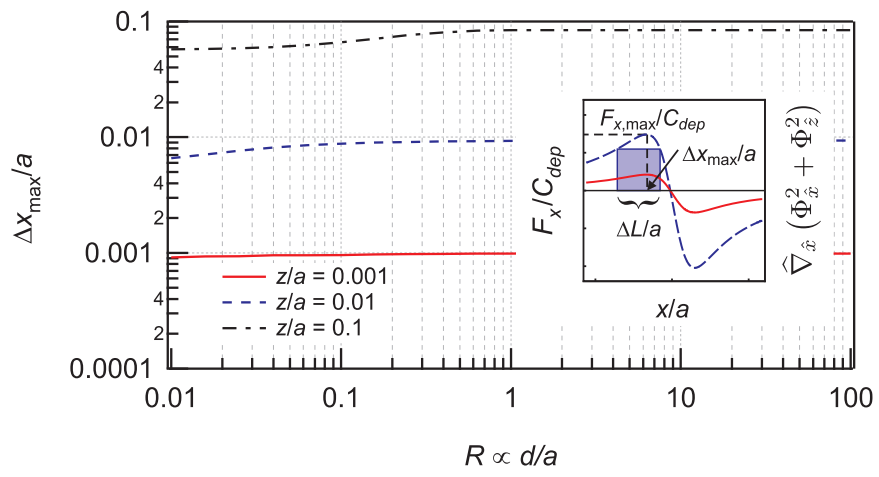

b) DEP force maximum position

FIG. 5. Dependence of the $x$-component of the positive DEP force maximum value normalized to $C_{d e p}$ (a) and the distance $\Delta x_{\max }$ of the point, where the $x$-component of the DEP force reaches its maximum value (b), from the domain wall (normalized to the domain spacing $a$ ) as a function of the parameter $R=(\pi d) /(2 a) \sqrt{\varepsilon_{a} / \varepsilon_{c}}$. The inset shows the maximum value of the DEP force $F_{x, \max } / C_{d e p}$ at the distance $\Delta x_{\max }$ from the domain wall and the distance $\Delta L$ over which the DEP force has a given minimal value. The parameters $\Delta x_{\max }$ and $\Delta L$ are measured as a fraction of domain spacing $a$.

is a dimensionless function given by the product of the effective value of the function $\left|\widehat{\nabla}_{\hat{x}}\left(\Phi_{\hat{x}}^{2}+\Phi_{\hat{z}}^{2}\right)\right|$ on the distance $\Delta L$ normalized to the domain spacing $a$, i.e. $\widehat{\Delta L}=k \Delta L$. For a given material, given values of the parameters $g$ and $R$, and a given distance from the surface of the ferroelectric film $\hat{z}$, it is possible to find a maximum value of the function $S_{\max }=\max \{S(\hat{z}, g, R)\}$ by a numerical calculation (see Tab. II).

\section{E. Conclusions}

The spatial distribution of the inhomogeneous electric field produced in a liquid above the top surface of the polydomain ferroelectric film has been calculated. The inhomogeneous electric field is the source of the electrophoretic and dielectrophoretic forces that can be used for a deposition of nanoparticle pattern. The key element of our analysis, considering the ionic nature of the liquid, makes our model quite realistic. Our numerical simulations have shown that the intersection of the domain wall with the surface of the ferroelectric film represents a trap for dielectric nanoparticles in the case of positive dielectrophoresis. The analysis of the critical conditions that control the deposition of dielectric nanoparticles and the stability of the deposited pattern are presented.

As an application of our analysis, one can consider two ways of realization of DEP force patterning using ferroelectric domains. First, it is the situation where the inhomogeneous electric field is produced by a spontaneous polarization reversal to form the required domain pattern using advanced methods of the nanoscale domain geometry engineering. Considering the values of material parameters that correspond to experiments by Grilli et $a .^{27}$, i.e. pentanoic acid with $\varepsilon_{m}=2.66$ and flour particles with $\varepsilon_{p}=3$, the estimated numerical parameters of positive DEP force are presented in Tab. II. It is
TABLE II. Calculated characteristic numerical parameters of the DEP force produced due to spontaneous polarization reversal from a single domain state to a polydomain pattern of a given geometry in the selected ferroelectric materials. It is considered: $r=1 \mu \mathrm{m}, z=0.01 a, R=10, \varepsilon_{m}=2.66$, $\varepsilon_{p}=3.0$, and $T=300 \mathrm{~K}$.

\begin{tabular}{llcccc}
\hline \hline Parameter & Unit & $\mathrm{LiTaO}_{3}$ & $\mathrm{LiNbO}_{3}$ & $\mathrm{BaTiO}_{3}$ & $\mathrm{PbTiO}_{3}$ \\
\hline$g$ & 1 & 18.1 & 18.6 & 309.3 & 34.0 \\
$C_{E}$ & $\mathrm{GV} \mathrm{m}^{-1}$ & 30 & 41 & 14 & 41 \\
$C_{\text {dep }}$ & $\mu \mathrm{N}$ & 168 & 312 & 37.5 & 321 \\
$\left|\nabla E^{2}\right|_{\max }$ & $10^{18} \mathrm{~V}^{2} \mathrm{~m}^{-3}$ & 110 & 195 & 0.09 & 60 \\
$F_{\text {dep }, \text { max }} / C_{\text {dep }}$ & $10^{-3}$ & 66 & 63 & 0.25 & 20 \\
$F_{\text {dep,rms }}$ & $\mu \mathrm{N}$ & 8.1 & 14.4 & 0.007 & 4.6 \\
$S_{\max }$ & $10^{-3}$ & 5.39 & 5.14 & 0.02 & 1.6 \\
$r_{\text {crit }}$ & $\mathrm{nm}$ & 1.5 & 1.2 & 15.8 & 1.8 \\
\hline \hline
\end{tabular}

seen that using this procedure, it is possible to achieve a large local values of the DEP forces that results in very small critical radii of particles (about $2 \mathrm{~nm}$ ) that can be trapped near the domain walls. Disadvantage of this method stems from the fact that the bound charges due to the discontinuous change of the spontaneous polarization at the surface of the ferroelectric film can be partially or completely compensated by free charge carriers due to the possible nonzero conductivity of the ferroelectric film or due to the ionic nature of the liquid. In this case, the inhomogeneous electrostatic field can disappear after some time and other method nanoparticle decoration should be used.

The second way of the realization of DEP forces using ferroelectric polydomain sample is the use of pyroelectric effect. It is known that the pyroelectric coefficients in the opposite domains are of the opposite sign. Therefore, when the ferroelectric polydomain sample is heated up 
TABLE III. Calculated characteristic numerical parameters of the DEP force produced due to pyroelectric effect after heating the ferroelectric film from the room temperature up by $\Delta T=50 \mathrm{~K}$ in the selected ferroelectric materials.

\begin{tabular}{|c|c|c|c|c|c|}
\hline Parameter & Unit & $\mathrm{LiTaO}_{3}$ & $\mathrm{LiNbO}_{3}$ & $\mathrm{BaTiO}_{3}$ & $\mathrm{PbTiO}_{3}$ \\
\hline$C_{E}$ & $\mathrm{GV} \mathrm{m}^{-1}$ & 0.62 & 0.11 & 0.54 & 1.1 \\
\hline$C_{d e p}$ & $\mathrm{nN}$ & 73.4 & 2.2 & 55.5 & 222 \\
\hline$\left|\nabla E^{2}\right|_{\max }$ & $10^{15} \mathrm{~V}^{2} \mathrm{~m}^{-3}$ & 48 & 1 & 0.1 & 40 \\
\hline$F_{d e p, \mathrm{rms}}$ & $\mathrm{nN}$ & 3.6 & 0.1 & 0.01 & 3.2 \\
\hline$r_{c r i t}$ & $\mathrm{~nm}$ & 20 & 64 & 140 & 20 \\
\hline
\end{tabular}

by $\Delta T$, the bound charges of the surface density $\pm \gamma_{S} \Delta T$ appear on the surface of the film due to the pyroelectric effect, where $\gamma_{S}$ is the pyroelectric coefficient and its sign differs from domain to domain. In order to get some numerical estimates for this case, the numerical values of parameters $C_{E}$ and $C_{d e p}$ should be calculated by replacing the symbol $P_{0}$ by $\gamma_{S} \Delta T$ in Eqs. $4 \mathrm{c}$ and $16 \mathrm{~b}$. Estimated numerical parameters of DEP forces achieved by this method are presented in Tab. III. Advantage of this approach is its relatively easy way of realization, e.g. using local laser beam heating of a single domain film or uniform heating of a polydomain film. Disadvantage is a presence of smaller values of DEP forces that result in greater value of the critical radius of particles that can be trapped at required locations (larger than $20 \mathrm{~nm}$ ). The numerical estimates presented in Tab. III are in a qualitative agreement with experimental results by Grilli et $a .^{27}$.

Finally, we have theoretically demonstrated that using ferroelectric domain patterns it is possible to achieve a very flexible way of nanoparticle decoration, which has several advantages: (i) using advanced and recently well developed methods of domain geometry engineering, it is possible to achieve a "rewritable" systems for the preparation of an arbitrary nanoparticle patterns, (ii) since the ferroelectric domain wall is an extremely thin spacial feature comparing with other ferroic material or deposited electrodes, it is possible to realize patterning of nanoparticles with sub-micron lateral dimensions. We believe that the obtained results represent a useful tool that can be profitably used for designing the systems with ferroelectric polydomain films representing a flexible way for decoration of nanoparticles.

\section{ACKNOWLEDGMENTS}

Authors would like to express their sincere gratitude to Štěpánka Klímková and Josef Šedlbauer for many useful discussions concerning the physical chemistry aspects of this work. This work has been supported by the Czech Science Foundation, Project Nos. GACR 202/06/0411, GACR 202/07/1289, and GACR P204/10/0616. Financial support of the Ministry of Education of the Czech
Republic (Grant no. MSM0021620835) is gratefully acknowledged.

${ }^{1}$ H. A. Pohl, J. Appl. Phys. 22, 869 (1951).

${ }^{2}$ H. A. Pohl, Dielectrophoresis: The Behavior of Neutral Matter in Nonuniform Electric Fields (Cambridge University Press, Cambridge, 1978).

${ }^{3}$ K. D. Hermanson, S. O. Lumsdon, J. P. Williams, E. W. Kaler, and O. D. Velev, Science 294, 1082 (2001).

${ }^{4}$ S. Banerjee, B. E. White, L. Huang, B. J. Rego, S. O'Brien, and I. P. Herman, J. Vac. Sci. Technol. B 24, 3173 (2006).

${ }^{5}$ A. Ramos, H. Morgan, N. Green, and A. Castellanos, J. Phys. D: Appl. Phys. 31, 2338 (1998).

${ }^{6}$ H. Morgan, M. Hughes, and N. Green, Biophys. J. 77, 516 (1999).

${ }^{7}$ P. R. C. Gascoyne and J. Vykoukal, Electrophoresis 23, 1973 (2002).

${ }^{8}$ T. B. Jones, M. Gunji, M. Washizu, and M. J. Feldman, J. Appl. Phys. 89, 1441 (2001).

${ }^{9}$ D. Lukas, A. Sarkar, and P. Pokorny, J. Appl. Phys. 103 (2008).

${ }^{10}$ M. P. Hughes, Nanotechnology 11, 124 (2000).

${ }^{11} \mathrm{~S}$. Matsumoto, E. Lim, H. Hertz, and M. Fejer, Electron. Lett. 27, 2040 (1991).

${ }^{12}$ M. Yamada, N. Nada, M. Saitoh, and K. Watanabe, App. Phys. Lett. 62, 435 (1993).

${ }^{13}$ S. Grilli, P. Ferraro, P. D. Natale, B. Tiribilli, and M. Vassalli, App. Phys. Lett. 87, 233106 (pages 3) (2005).

${ }^{14}$ O. Kolosov, A. Gruverman, J. Hatano, K. Takahashi, and H. Tokumoto, Phys. Rev. Lett. 74, 4309 (1995).

${ }^{15}$ A. Gruverman, O. Auciello, and H. Tokumoto, Annu. Rev. Mater. Sci. 28, 101 (1998).

${ }^{16}$ X. Liu, K. Terabe, and K. Kitamura, Jpn. J. Appl. Phys. 44, $7012(2005)$.

${ }^{17}$ B. J. Rodriguez, R. J. Nemanich, A. Kingon, A. Gruverman, S. V. Kalinin, K. Terabe, X. Y. Liu, and K. Kitamura, App. Phys. Lett. 86, 012906 (pages 3) (2005).

${ }^{18}$ Y. Cho, K. Fujimoto, Y. Hiranaga, Y. Wagatsuma, A. Onoe, K. Terabe, and K. Kitamura, App. Phys. Lett. 81, 4401 (2002).

${ }^{19}$ J. Son, Y. Yuen, S. Orlov, and L. Hesselink, J. Cryst. Growth 281, 492 (2005).

${ }^{20}$ X. Li, K. Terabe, H. Hatano, and K. Kitamura, Jpn. J. Appl. Phys. 44, L1550 (2005).

${ }^{21}$ D. Li, D. Strachan, J. Ferris, and D. Bonnell, J. Mater. Res. 21, 935 (2006).

${ }^{22}$ D. Li and D. A. Bonnell, Annu. Rev. Mater. Res. 38, 351 (2008).

${ }^{23} \mathrm{~S}$. Kalinin, D. Bonnell, T. Alvarez, X. Lei, Z. Hu, R. Shao, and J. Ferris, Adv. Mater. 16, 795 (2004).

${ }^{24}$ D. Li and D. A. Bonnell, Ceram. Int. 34, 157 (2008).

${ }^{25}$ S. Dunn, D. Cullen, E. Abad-Garcia, C. Bertoni, R. Carter, D. Howorth, and R. W. Whatmore, App. Phys. Lett. 85, 3537 (2004).

${ }^{26}$ C. Ke, X. Wang, X. P. Hu, S. N. Zhu, and M. Qi, J. Appl. Phys. 101, 064107 (pages 6) (2007).

${ }^{27}$ S. Grilli and P. Ferraro, App. Phys. Lett. 92, 232902 (2008).

${ }^{28}$ P. Debye and E. Hückel, Physikalische Zeitschrift 24, 185-206 (1923).

29 A. Kopal, T. Bahník, and J. Fousek, Ferroelectrics 202, 267 (1997).

${ }^{30}$ A. Savage, J. Appl. Phys. 37, 3071 (1966).

${ }^{31}$ D. A. Scrymgeour, V. Gopalan, A. Itagi, A. Saxena, and P. J. Swart, Phys. Rev. B 71, 184110 (2005).

${ }^{32}$ R. W. Whatmore, Rep. Prog. Phys. 49, 1335 (1986).

${ }^{33}$ A. G. Chynoweth, J. Appl. Phys. 27, 78 (1956).

${ }^{34}$ Y. L. Li, L. E. Cross, and L. Q. Chen, J. Appl. Phys. 98, 064101 (pages 4) (2005).

${ }^{35}$ M. J. Haun, E. Furman, S. J. Jang, H. A. McKinstry, and L. E. Cross, J. Appl. Phys. 62, 3331 (1987).

${ }^{36}$ M. Washizu, S. Suzuki, O. Kurosawa, T. Nishizaka, and T. Shinohara, IEEE Trans. Ind. Appl. 30, 835 (1994).

${ }^{37}$ M. P. Hughes and H. Morgan, J. Phys. D: Appl. Phys. 31, 2205 (1998). 